\title{
To Assess the Effectiveness of Self-Instructional Mockule on Knowledge regarding Effect of Massage Therapy in Reduaing Labor Pain
}

\author{
${ }^{1}$ Simer Preet Kaur, ${ }^{2}$ Sudha A Raddi \\ ${ }^{1}$ Assistant Professor, Sumandeep College of Nursing, Sumandeep Vidyapeeth University, Piparia, Vadodara, Gujarat, India \\ ${ }^{2}$ Principal, Professor and Head, Department of Obstetrics and Gynecology, KLEUs Institute of Nursing Sciences, Belgaum, Karnataka, India
}

Correspondence: Simer Preet Kaur, Assistant Professor, Sumandeep College of Nursing, Sumandeep Vidyapeeth University Piparia, Vadodara-391760, Gujarat, India, P hone: 09601930067, e-mail: kaur.simerpreet@ gmail.com

\section{ABSTRACT}

Objectives: To assess the knowledge of 3rd year GNM students regarding effect of massage therapy in reducing labor pain before and after the implementation of self-instructional module. To evaluate the effect of self-instructional module in enhancing the knowledge of 3 rd year GNM students regarding the effect of massage therapy in reducing labor pain and to compare the knowledge of samples regarding the effect of massage therapy in reducing labor pain in relation to selected demographic variables (age at 12th std. education, medium of 12 th std. education, percentage of 1st and 2nd year GNM exam, number of attempts, location of house and mother education).

Methods: The research approach for the study was that of an evaluative one with one group pre- and post-test design. The sample size considered for the study was 50 general nursing and midwifery students. The sampling technique used for the study was convenience sampling, which is a type of nonprobability sampling. The tool used for gathering relevant data was a structured questionnaire on knowledge of effect of massage therapy in reducing labor pain.

Results: Analysis of data was done on the basis of objectives and hypotheses. Data analysis of level of knowledge revealed that during pretest, no student had highly adequate knowledge of effect of massage therapy on labor pain, $9(18 \%)$ had moderately adequate knowledge, while $41(82 \%)$ had inadequate knowledge. It was worth noting during post-test, where no student had inadequate knowledge and majority of $50(100 \%)$ had highly adequate knowledge. While assessing the effectiveness of the structured instructional module on effect of massage therapy on labor pain, the pre-test and post-test data analysis by using Wilcoxon signed rank test revealed the mean post-test score (27.88 $\pm 1.27)$ was higher than the mean pre-test score $(13.14 \pm 2.93)$. Since the calculated $z$-value was 6.16 and $p$-value was 0.00 , thus proving that $p<0.05$ (data was analyzed using SPSS software).

Conclusions: Based on the analyses, the following inference was drawn. There was evident increase in the knowledge scores in all the areas included in the study after the administration of self-instructional module. Thus, it was inferred that the structured teaching program was effective and while the knowledge gain score is commendable, there is still room for improvement.

Keywords: General nursing and midwifery (GNM) students, Knowledge, Self-instructional module, Massage therapy, Labor.

\section{INTRODUCTION}

Pain is unpleasant, but most useful sensation developed early in evolution as a protective mechanism. A dvancement in science and technology has developed. Some form of analgesia, which is always available, few women are brain washed into equating pain relief in labor with personal failure. ${ }^{1} \mathrm{M}$ ore often than not, these women not only suffer needlessly, but also reduce their chance of normal delivery. U nrelieved maternal pain leads to a series of metabolic changes in the mother, which may adversely affect the fetus. D uring the first stage of labor, women usually perceive the visceral pain of diffuse abdominal cramping and uterine contractions. In the second stage of labor, there is a sharper and more continuous somatic pain in the perineum. Pressure or nerve entrapment caused by the fetus's head can cause severe back or leg pain. ${ }^{2}$

Nulliparous women generally experience more sensory pain during early labor while multiparous women experience more intense pain during the late first stage and the second stage of labor as a result of rapid fetal descent. Pregnant women benefit from massage therapy. If we consider the benefits and possible side effects of other nonpharmacological methods, massage is a method which will not produce any harmful effects to the mother as well as the fetus. ${ }^{3} \mathrm{M}$ assage is an art of touch with just the bare human hands, which nevertheless involves coordination of high order and use of great skill. M assage stimulates the body to release endorphins, which are natural pain-killing and mood-lifting substances. Endorphins are responsible for the "feel-good" factor. ${ }^{4}$

Today's scenario demonstrates that there is a growing demand for natural birth and use of nonpharmacological methods for pain relief during labor. In this way, complementary and alternative therapies based on the philosophy of holism, and an appreciation of the innate interaction between body, mind and spirit of the individual are spreading its root. Technology 
and medical care, with all its benefits, cannot substitute for the compassionate touch of human beings. H umans are communal people who fail to thrive without touch. M others need healing touch to give birth to healthy babies and raise healthy children. Babies need touch to become healthy adults and parents. The touch of a midwife to a mother and of a mother to her child is vital link for developing physical and emotional health in a human being. ${ }^{5}$

\section{METHODS}

Research approach was descriptive evaluative approach and pre-experimental, one group pre- and post-test design was used. The data was collected using close-ended questionnaire, and sampling method used was convenience sampling method, which is one of the nonprobability sampling method. The investigator personally contacted each sel ected subject and the informed written consent was obtained after explaining the purpose of the study. Pretest was conducted on 2 July 2008, followed by administration of structured instructional module, and post-test was conducted after 5 days.

\section{RESULTS}

The findings of the study showed that $43(86 \%)$ students were in age group $\leq 22$. Distribution of 12 th std. education revealed that 32 (64\%) were from arts group. The distribution of medium of 12 th std. education reported 34 (68\%) have done in M arathi medium. Religion depicted 40 (80\%) belonged to Hinduism. L ocation of house depicted 24 (48\%) were from urban areas. Distribution of attempts in 1st year GNM exam revealed that $23(46 \%)$ passed in 1st attempt. R egarding percentage of marks obtained in 1st year GN M 32 (64\%) obtained marks between 60 and 74\%. Distribution of attempts in 2nd year, GN M exam revealed 27 (54\%) passed in 2 nd attempt. R egarding percentage of marks obtained in 2nd year G N M , 42 (84\%) obtained marks

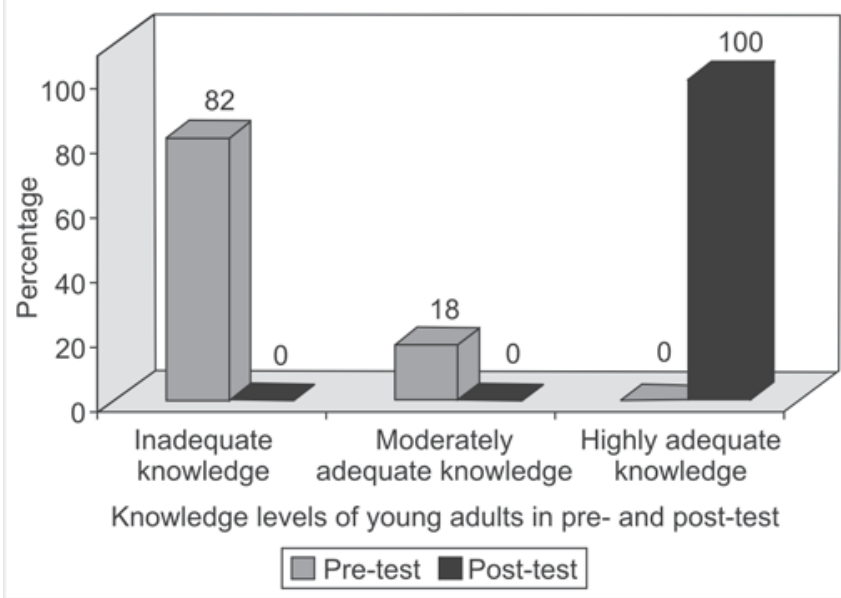

Graph 1: Percentage-wise distribution of students by their overall knowledge on effect of massage therapy in reducing labor pain in pre-test and post-test between 60 and 74\%. M ajority of mothers of students 15 (30\%) were educated up to secondary. R egarding previous education in al ternative therapies, 50 (100\%) students did not receive any education or training. Pretest revealed that no student had highly adequate knowledge of effect of massage therapy on labor pain, $9(18 \%)$ had moderately adequate knowledge and $41(82 \%)$ had inadequate knowledge. It was worth noting that no student had inadequate knowledge and majority of 50 (100\%) had highly adequate knowledge (Graph 1 ). While assessing the effectiveness of the structured instructional module on effect of massage therapy on labor pain, the pre- and post-test data analysis using Wilcoxon signed rank test revealed the mean post-test score $(27.88 \pm 1.27)$ was higher than the mean pretest score (13.14 \pm 2.93 ) (Graph 2). Since the calculated z-value was 6.16 and $p$-value was 0.00 , thus proving that $p<0.05$. Distribution of student with regard to their knowledge on various aspects of massage therapy during labor before and after the implementation of SIM showed that the students had very less know ledge regarding the location of massage given to mother during labor $6(12 \%)$ and the peak period for providing massage therapy during labor 9 (18\%) (Table 2) (data was analyzed by using SPSS Software).

\section{DISCUSSION}

In pretest, $82 \%$ of the students had inadequate knowledge and remaining $18 \%$ of the students had moderately adequate knowledge. No student had highly adequate knowledge during pretest. Post-test scores compared to pretest scores showed an observable increase in the knowledge of students as $100 \%$ of them had highly adequate knowledge and no student had moderately adequate or inadequate knowledge regarding effect of massage therapy in reducing labor pain. This finding is supported by the study done by M elzack $\mathrm{R}$ et al, which focused on the meaning of nursing presence during labor and birth to Canadian childbearing women showed that laboring women value physical presence, emotional support and advocacy. ${ }^{6}$ Chang et al conducted a randomized controlled study to assess the effects of massage on pain and anxiety during labor among primi mother at regional hospital s in Southern Tai wan. ${ }^{7}$ A round

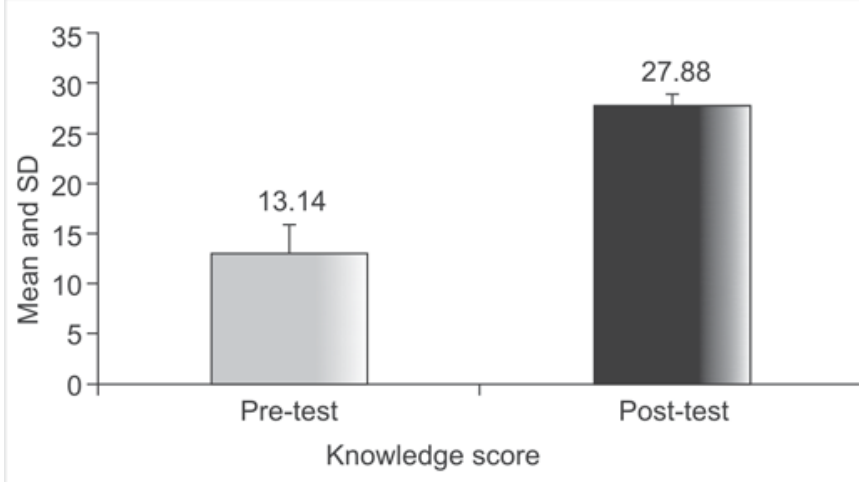

Graph 2: Comparison of knowledge level of 3rd year GNM students to evaluate the effectiveness of self-instructional module on effect of massage therapy in reducing labor pain 
To Assess the Effectiveness of Self-Instructional Module on Knowledge regarding Effect of Massage Therapy

60 primiparous mothers were chosen by random method and assigned to either the experimental $(n=30)$ the control $(n=30)$ group. The nurse rated present behavioral intensity as a measure of labor pain. VAS for anxiety was used to collect the data. The study results showed that the anxiety levels were only significantly different in two groups. A total of 26 out of 30 $(87 \%)$ in experimental group reported that massage therapy was helpful to reduce labor pain and anxiety. For this, knowledge should be there regarding massage therapy during labor. Zang et al conducted a descriptive study to assess the psychological nursing to ease labor pains among women in selected hospitals of USA ${ }^{8} \mathrm{H}$ undred laboring women were randomly assigned to psychological support group $(n=50)$ and control group $(n=$ 50). V isual analog scale was used to assess the pain. The study result showed that the pain scores of the psychological support group was lower (0.01) than the control group (0.03) in the first stage of labor. The time of the total labor in the psychological support group was shorter than that of the control group $(p<0.001)$. A study on alternative medicine therapies, knowledge and attitude of health professionals concludes that majority of nurses had good knowledge regarding the use of al ternative medicine in nursing practice; $24 \%$ reported that formal nurses practitioner education was source of knowledge and $60 \%$ reported that their personal experience had improved their knowledge regarding alternative medicine. It is supported by the A manda B askwill et al systemic review, which showed that $76.4 \%$ of obstetricians and $91.1 \%$ of midwives who responded though massage was useful (when asked to choose between useful, not useful or no opinion). A dditionally, $64.1 \%$ and $57.5 \%$ of obstetricians and midwives respectively had referred patients for massage therapy. When asked about the perceived safety of massage during pregnancy, $77.9 \%$ of obstetricians and $94.8 \%$ of midwives answered massage was safe. ${ }^{9}$

\section{CONCLUSIONS}

A fter the detailed analysis, this study leads to the following conclusions:

Students did not have $100 \%$ knowledge regarding effect of massage therapy in reducing labor pain (Table 1). They require further education because all of them need to enhance their knowledge. There was a significant increase in the knowledge of subjects after the introduction of SIM (Table 2). To find the effectiveness of SIM, Wilcoxon signed rank test was applied and z-value was calculated, post-test score was significantly higher at 0.05 levels than that of pretest score. Thus, it is concluded that SIM on effect of massage therapy in reducing labor pain was found effective as a teaching strategy.

Knowledge of the students was divided into three levels, inadequate, moderately adequate and highly adequate knowledge. Post-test scores compared to pretest scores showed an observable increase in the knowledge of students as $100 \%$ of them had highly adequate knowledge and no student had moderately adequate or inadequate knowledge regarding effect of massage therapy in reducing labor pain. Demographic variables do not show a major role in influencing students post-test knowledge score. Only age of the students was found significant, thus influencing their post-test knowledge.

Hence, on the above cited findings, it could be concluded undoubtedly that the written material prepared by the investigator in the form of SIM helped the students to improve their knowledge on effect of massage therapy in reducing labor

Table 1

Percentage-wise distribution of overall knowledge level of students in pre- and post-test

\begin{tabular}{|lccccc}
\hline & \multicolumn{2}{c}{ Pre-test } & & \multicolumn{2}{c}{ Post-test } \\
\cline { 2 - 4 } & Frequency & Percentage & & Frequency & Percentage \\
\hline Inadequate knowledge & 41 & 82.00 & 0 & 00.00 \\
Moderately adequate knowledge & 9 & 18.00 & 0 & 00.00 \\
Highly adequate knowledge & 0 & 00.00 & 50 & 100.00 \\
\hline
\end{tabular}

Table 2

Distribution of students with regard to their knowledge on massage therapy during labor before and after the implementation of SIM

\begin{tabular}{|c|c|c|c|c|}
\hline \multirow[t]{2}{*}{ Knowledge area } & \multicolumn{2}{|c|}{ Pre-test } & \multicolumn{2}{|c|}{ Post-test } \\
\hline & Frequency & Percentage & Frequency & Percentage \\
\hline Location of massage given to mother during labor & 6 & 12 & 49 & 98 \\
\hline Peak period for providing massage therapy during labor & 9 & 18 & 43 & 86 \\
\hline Massage therapy during labor helps to reduce & 24 & 48 & 48 & 96 \\
\hline Midwife can practice massage therapy & 37 & 74 & 49 & 98 \\
\hline Massage therapy helps mother to relax during contractions & 33 & 66 & 50 & 100 \\
\hline Massage therapy produces harmful effect on fetus & 34 & 68 & 50 & 100 \\
\hline Massage during labor helps in reducing postpartum depression & 21 & 42 & 49 & 98 \\
\hline
\end{tabular}


pain. Thus, they can apply their knowledge in clinical and community settings.

\section{REFERENCES}

1. Kara S M aia. Touch in the M idwifery arts. M idwifery Today. Jan 2005;8(4):271-73.

2. Kabeyama K, M iyoshi M . Longitudinal Study of the Intensity of memorized L abour Pain. Journal of N ursing Practices (online) Feb 2001;7(1):46-53.

3. SylviaT Brown, Carol Douglas, LeeA nn P. W omen's Evaluation of Intrapartum Non pharmacological Methods used during L abour. J ournal of Perinatal Education 2001 (online) Summer, 10(3):1-8.

4. Hormones during labour and birth. www. perinatal education associates. In; 2007.
5. K elly Lott. http://www.pregnancytoday.com/. The art of $M$ assage during $L$ abour.

6. M elzack R, K inch R, D obkin P, L ebrun M, T aenzer P. Severity of labour pain: Influence of physical as well as psychological variables; Canadian M edical A ssociation J ournal (online) M ar 1984;130(5):579-84.

7. Chang MY. Effects of massage on pain and anxiety during labour: A randomized controlled trial in Taiwan. Journal of A dvance Nursing (online) 2002;38(1):68-73.

8. Zhang $C L, Y u Z J$, Feng $A H$. Psychological Nursing to Ease Pain during Labour (online) J une 1996;31(6):311-13.

9. A manda Baskwill, T rish D ryden. Literature review on pregnancy and massage. O ntario M assage Therapist A ssociation and Centennial College, Centre for Applied Research in Health. (online) 2005. 